\title{
Editorial
}

\section{Chronic Periodontal Inflammation: The Cellular Human Time Bomb}

Inflammation is the first response of the immune system to infection or irritation and may be referred to as the innate cascade. Inflammation is characterized by the following quintet: redness, heat, swelling, pain and dysfunction of the organs involved inflammation has two main componentscellular and exudative. Inflammation influences the physiology, biochemistry, cell biology and pharmacology of human body. Inflammation extends to acute and chronic inflammation; mediators of inflammation; mechanisms, cytotoxicity and pharmacology of tissue injury.

The concept of inflammation is long recognized. Immune responses to injury and infection are essential; however, they cause problems when inflammatory processes are maladaptive, leading to

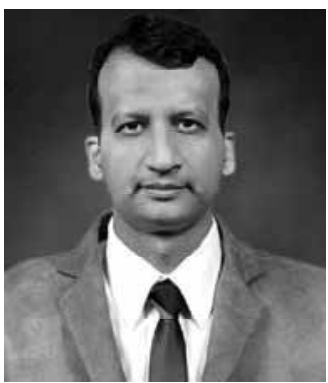
chronic diseases, such as diabetes and chronic heart diseases. Scientific research is needed to expound the complex pathways involved in low grade chronic inflammation. Obviously, numerous mechanisms are involved; eventually, the question is how these can be used to superior diagnose or treat the late-stage sequelae that many lines of verification argue are driven by inflammatory processes.

The pathogenesis of periodontal disease is an inflammatory process involving innate and adaptive immune responses. Periodontal disease is characterized by the host-mediated destruction of soft tissue caused by the induced production and activation of lytic enzymes and stimulated osteoclastogenesis. The inflammatory progression occurring in periodontal disease is characterized by the infiltration of leukocytes, which limit the level of bacterial invasion. There are paramount number of aspects that promote leukocyte recruitment, including bacterial products, cytokines, cross-talk between innate and adaptive immune responses, chemokines, lipid mediators and complement. An intermediary system that lies between bacterial stimulation and tissue injury is the production of cytokines, which stimulates inflammatory events that activate effector mechanisms. These cytokines can be organized as chemokines, innate immune cytokines and acquired immune cytokines. Although they were historically identified as leukocyte products, many are also produced by a number of cell types, including keratinocytes, resident mesenchymal cells (such as fibroblasts and osteoblasts) or their precursors, dendritic cells and endothelial cells. Chemokines are chemotactic cytokines that play an important role in leukocyte recruitment and may directly or indirectly modulate osteoclast formation.

The periodontium responds to oral biofilm by the process of inflammation. Dental biofilm release a variety of biologically active products, such as bacterial lipopolysaccharides (endotoxins), chemotactic peptides, protein toxins and organic acids. These molecules stimulate the host to generate a multiplicity of responses, among them the production and release of potent agents known as cytokines. These include interleukin-1 beta (IL-1), interleukin-8 (IL-8), prostaglandins (PGE2) and tumor necrosis factor-alpha (TNF- $\alpha)$. There is a broad range of periodontal response to these molecules, from mild gingivitis to severe destructive periodontitis.

Periodontal inflammatory responses are multifaceted and consist of varied elements. Immediate-type responses including vasodilatation, increased vascular permeability and leukocyte extravasation, are mediated by endogenous mediators, including prostanoids, kinins and neuropeptides. Nonspecific immune responses, including polymorphonuclear leukocyte and monocyte migration and activation, and cytokine production, are elicited in response to bacteria and their products. Interleukin-1 and prostaglandins in particular have been implicated as central mediators of periodontal bone resorption. Chronic periodontal inflammation further involves specific T- and B-cell-mediated antibacterial responses and activates a network of regulatory cytokines which are produced by Th 1- and Th2-type T-lymphocytes.

The role of polymorphonuclear (PMN) leucocytes is the principally the protective one, host tissue injury can result indirectly from over exuberant PMN and monocytes response. Polymorphonuclear generates the reactive oxygen species (ROS) superoxide through respiratory burst as a part of host reaction to infection. Patients with periodontal disease demonstrate increased PMN number and activity. It has been observed that this proliferation results in a high degree of ROS release resulting in extensive oxidative damage to periodontal tissues.

Control of periodontal diseases is most effectively performed by eliminating or suppressing the organisms that cause them and establishing a host compatible microbiota to prevent the initiation of inflammatory-immune cascade of reactions.

Recent research evidence proved that periodontal infection significantly enhances the risk for certain systemic diseases or alter the natural course of systemic conditions. Thus, the control of low grade chronic inflammation of periodontal infection will be 'Game Changer' in the oral and systemic health.

Rajiv Saini

Editor-in-Chief 